\title{
Modeling size-dependent behaviors of axially functionally graded Bernoulli-Euler micro-beam
}

\author{
Shuai WANG, Zetian KANG, Shichen ZHOU, Bo ZHOU*, Shifeng XUE \\ College of Pipeline and Civil Engineering, China University of Petroleum (East China), Qingdao 266580, P. R. China
}

*Corresponding Author: Bo ZHOU, Email: zhoubo@upc.edu.cn

\begin{abstract}
:
This work focus on the mechanical behaviors, which are related to the size effect, functionally graded (FG) effect and Poisson effect, of an axially functionally graded (AFG) micro-beam whose elastic modulus varies according to sinusoidal law along its axial direction. The displacement field of the AFG micro-beam is set according to the Bernoulli-Euler beam theory. Employing the modified couple stress theory (MCST), the components of strain, curvature, stress and couple stress are expressed by the second derivative of the deflection of the AFG micro-beam. A size-dependent model related to FG effect and Poisson effect, which includes the formulations of bending stiffness, deflection, normal stress and couple stress, is developed to predict the mechanical behaviors of the AFG microbeam by employing the principle of minimum potential energy. The mechanical behaviors of a simply supported AFG micro-beam are numerically investigated using the developed model for demonstrating the size effects, FG effects and Poisson effects of the AFG micro-beam. Results show that the mechanical behaviors of AFG micro-beams are distinctly size-dependent only when the ratio of micro-beam height to material length-scale parameter is small enough. The FG parameter is an important factor that determines and regulates the size-dependent behaviors of AFG micro-beams. The influences of Poisson's ratio on the mechanical behaviors of AFG micro-beams are not negligible, and should be also considered in the design and analysis of an AFG micro-beam. This work supplies a theoretical basis and a technical reference for the design and analysis of AFG micro-beams in the related regions.

Keywords: axially functionally graded micro-beam; size-dependent behaviors; size effect; functionally graded effect; Poisson effect
\end{abstract}

\section{Introduction}

Functionally graded (FG) materials are a new group of non-homogeneous materials and have some desirable performances to satisfy special features in engineering design ${ }^{[34]}$. By proper design, they can not only eliminate the stress concentration, reduce the residual stress, but also reduce crack driving force to improve the strength of structural members. In the last decade, they are commonly used in applications of construction, aerospace, energy absorption or even in biomedical sectors [26]. Many theoretical researches on the macroscopical characteristics of FG materials have been carried out based on the classical continuum mechanics ${ }^{[6,21,22]}$.

With the rapid development of micro-technologies, FG materials have been broadly applied in micro-electromechanical system (MEMS) and nano-electro-mechanical system (NEMS). Micro-scale experiments [13-14] have revealed the size-dependent properties of micro-structures, which is called size effect ${ }^{[33]}$. The classical continuum theory fails to express the size effects of microstructures.
Therefore, several higher-order continuum mechanics theories, such as strain gradient theories, couple stress theory and nonlocal elasticity theory, have been proposed to capture the size effects in microstructures ${ }^{[28]}$. In the couple stress theory, the size effects of microstructures are interpreted by two material length-scale parameters. The modified couple stress theory (MCST), which contains one material length-scale parameter, has been proposed by Yang et al. (2002) $)^{[32]}$ and widely used to investigate the size-dependent bending, vibration, dynamic and buckling behaviors of the microstructures made of homogeneous materials $[2-4,12,17-18,20]$.

In recent years, the mechanical behaviors of transverse functionally graded (TFG) micro-beams/plates, whose material properties vary along the thickness direction, have been investigated on the basis of MCST. Asghari et al. (2010) ${ }^{[1]}$ investigated the size effects of static bending deflection and natural frequency of a TFG cantilever micro-beam using the Bernoulli-Euler beam theory and Hamilton's principle. Reddy (2011) ${ }^{[23]}$ developed nonlinear Bernoulli-Euler and Timoshenko micro-beam theories, 
which can consider the Poisson effect and the von Kármán geometric nonlinearity, to study the size effects and FG effects of bending deflection, natural frequency and buckling of a simply supported TFG micro-beam based on the principle of virtual displacements. Ke et al. (2011,2012a, $2012 b)^{[9-11]}$ investigated the size effects and FG effects of bending, free vibration and buckling characteristics of TFG composite micro-beams/plates with different boundary conditions using the Hamilton's principle. Salamat-Talab et al. (2012) ${ }^{[24]}$ analyzed the size effects, FG effects and Poisson effects of the deflection, rotation and natural frequencies of a simply supported TFG micro-beam utilizing a third-order shear deformation beam theory, Hamilton's principle and series method. Thai et al. (2015) [27] studied the size effects and FG effects of the normal stress, transverse shear stress, deflection and frequency of a simply supported TFG sandwich Timoshenko microbeam based on the Hamilton's principle and Mori-Tanaka scheme. And then, considering the Poisson effect, Trinh et al. (2016) ${ }^{[29]}$ used a unified beam theory to explore the influences of the material length-scale parameter, FG parameter and slenderness ratio on the deflection, stresses, natural frequencies and critical buckling loads of a simply supported TFG micro-beam.

In addition to these TFG micro-beams/plates aforementioned, axially functionally graded (AFG) micro-beams, whose material properties vary along the longitudinal direction, have also been investigated ${ }^{[7,25,30]}$. For instance, Ghayesh et al. (2017) ${ }^{[5]}$ examined the size effects of the nonlinear bending and forced vibrations of an AFG Bernoulli-Euler tapered micro-beam, and the influences of FG parameter and taper ratio on the deflection and frequency based on the MCST, Hamilton's principle, Galerkin method and Newton-Raphson technique. Li et al. (2017) ${ }^{[16]}$ studied the size effects and FG effects of the bending, bucking and free vibration of an AFG Bernoulli-Euler micro-beam using the nonlocal strain gradient theory, the Hamilton's principle and generalized differential quadrature method. Karamanl 1 and Vo (2018 ${ }^{[8]}$ investigated the size-dependent flexural behavior of a bi-directional FG micro-beam based on the MCST and the principle of minimum potential energy for several boundary conditions.

All of size effect, FG effect and Poisson effect play important roles in governing the mechanical behaviors of both TFG and AFG micro-beams. However few work have been published to investigate all of size effects, FG effects and Poisson effects of an AFG micro-beam comprehensively. The objective of this paper is to develop the mechanical model of an AFG micro-beam, which is related to the size effects, FG effects and Poisson effects. Firstly the displacement field of an AFG micro-beam is set according to the Bernoulli-Euler beam theory, and the components of strain, curvature, stress and couple stress are expressed by the deflection of the AFG micro-beam in Section 2. And then an AFG micro-beam model, which includes the size-dependent formulations of bending stiffness, deflection, stress and couple stress of the AFG micro-beam, is established by employing the principle of minimum potential energy in Section 3. Subsequently the size effects, the FG effects and the Poisson effects of the AFG micro-beam are respectively investigated in Section 4, Section 5 and Section 6 based on the developed model. Finally the important conclusions related to the size-dependent behaviors of an AFG micro-beam are summarized in section 7 .

\section{MCST descriptions of basic variables}

According to the MCST, a generic matter point in an elastomer has 6 degrees of freedom, including 3 displacement components expressed by the vector $u_{i}$ and 3 rotation components expressed by the vector $\theta_{i}$, respectively. The differential relationship between displacement vector and rotation vector reads as

$\theta_{i}=\frac{1}{2} e_{i j k} u_{k, j}$

where $e_{i j k}$ is the permutation symbol. The geometric equations of an elastomer read as

$\varepsilon_{i j}=\frac{1}{2}\left(u_{i, j}+u_{j, i}\right)$

$\chi_{i j}=\frac{1}{2}\left(\theta_{i, j}+\theta_{j, i}\right)$

where $\varepsilon_{\mathrm{ij}}$ and $\chi_{\mathrm{ij}}$ denote the strain tensor and curvature tensor, respectively. For the case of small deformation, we write the constitutive equations of an isotropic elastomer as $\sigma_{i j}=\lambda \varepsilon_{k k} \delta_{i j}+2 G \varepsilon_{i j}$

$m_{i j}=2 l^{2} G \chi_{i j}$

where $\sigma_{\mathrm{ij}}$ is the stress tensor, $\mathrm{m}_{\mathrm{ij}}$ is the deviatoric part of couple stress tensor, which is shortly called as the couple stress tensor in this paper, 1 is called as the material lengthscale parameter, which is a material constant characterizing the size effect, $\lambda$ and $G$ represent Lame's coefficients expressed as

$$
\begin{aligned}
& \lambda=\frac{E \mu}{(1+\mu)(1-2 \mu)} \\
& G=\frac{E}{2(1+\mu)}
\end{aligned}
$$

with $\mathrm{E}$ and $\mu$ being the elastic modulus and Poisson's ratio, respectively.

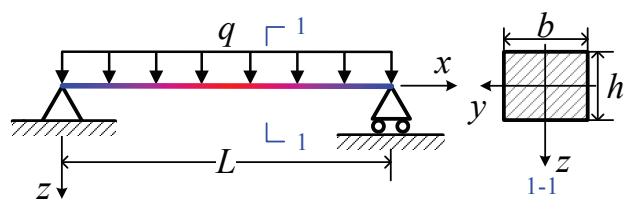

Figure 1 Simply supported AFG micro-beam subjected to uniformly distributed load under a rectangular coordinate system.

Figure 1 plots a simply supported AFG microbeam with rectangular constant section subjected to the uniformly distributed load under a rectangular coordinate system. The length, width and thickness are respectively presented by $\mathrm{L}, \mathrm{b}$ and $\mathrm{h}$. Its elastic modulus is assumed to vary along the axial direction according to 


$$
E(x)=E_{0}+\left(E_{1}-E_{0}\right) \sin \frac{\pi x}{L}
$$

where $\mathrm{E}_{0}$ and $\mathrm{E}_{1}$ stand for the values of elastic modulus in the left end and the middle cross-section of the microbeam, respectively. According to the Bernoulli-Euler beam theory, we can express the displacement field of the AFG micro-beam as

$u=-z \frac{\mathrm{d} w}{\mathrm{~d} x}, v=0, w=w(x)$

where $\mathrm{u}, \mathrm{v}$ and $\mathrm{w}$ are the displacement components in the $\mathrm{x}-, \mathrm{y}$ - and $\mathrm{z}$ - directions, respectively. The displacement component in $\mathrm{z}$-direction, $\mathrm{w}$, is often called as the deflection of beam. Substituting the displacement field Eq. (6), into the geometric equation Eq. (2a), we formulate the strain components of the AFG micro-beam as

$\varepsilon_{x}=-z \frac{\mathrm{d}^{2} w}{\mathrm{~d} x^{2}}, \varepsilon_{y}=\varepsilon_{z}=0$

$\varepsilon_{x y}=\varepsilon_{y x}=\varepsilon_{z x}=0$

Substituting the displacement field Eq. (6) into the rotation components Eq. (1), we formulate the rotation components of the AFG micro-beam as

$\theta_{y}=-\frac{\mathrm{d} w}{\mathrm{~d} x}, \theta_{x}=\theta_{z}=0$

Plugging the rotation components Eq. (8) into the geometric equation Eq. (2b), we formulate the curvature components of the AFG micro-beam as

$\chi_{x x}=\chi_{y y}=\chi_{z z}=0$,

$\chi_{x y}=\chi_{y x}=-\frac{1}{2} \frac{\mathrm{d}^{2} w}{\mathrm{~d} x^{2}}, \chi_{x z}=0$

Plugging the strain components Eq. (7) into the constitutive equation Eq. (3a), we formulate the stress components of the AFG micro-beam as

$\sigma_{x x}=-[\lambda(x)+2 G(x)] z \frac{\mathrm{d}^{2} w}{\mathrm{~d} x^{2}}$,

$\sigma_{z z}=\sigma_{y y}=-\lambda(x) z \frac{\mathrm{d}^{2} w}{\mathrm{~d} x^{2}}$,

$\sigma_{x y}=\sigma_{y z}=\sigma_{z x}=0$

where the coefficients $\lambda(\mathrm{x})$ and $\mathrm{G}(\mathrm{x})$ can be determined by Eqs. (5) and (4). Plugging the curvature components Eq. (9) into the constitutive equation Eq. (3b), we formulate the couple stress components of the AFG micro-beam as

$m_{x x}=m_{y y}=m_{z z}=0$,

$m_{x y}=m_{y x}=-G(x) l^{2} \frac{\mathrm{d}^{2} w}{\mathrm{~d} x^{2}}$

\section{AFG micro-beam model}

\subsection{Bending stiffness formulation}

According to the MCST, we can calculate the strain energy of an elastomer by

$U=\frac{1}{2} \int_{V}\left(\sigma_{i j} \varepsilon_{i j}+m_{i j} \chi_{i j}\right) \mathrm{d} V$

where $\mathrm{V}$ is the volume the elastomer occupying. Inserting the strain Eq. (7), curvature Eq. (9), stress Eq. (10) and couple stress Eq. (11) into the strain energy Eq.
(12), we obtain

$U=\frac{1}{2} \int_{L} K(x)\left(\frac{\mathrm{d}^{2} w}{\mathrm{~d} x^{2}}\right)^{2} \mathrm{~d} x$

where

$K(x)=\alpha\left[E_{0}+\left(E_{1}-E_{0}\right) \sin \frac{\pi x}{L}\right] I$

is the bending stiffness of the AFG micro-beam, where $I=\int_{A} z^{2} \mathrm{~d} A$

is the inertia moment of the cross-section of the micro-beam, and

$\alpha=\frac{(1-\mu)}{(1-2 \mu)(1+\mu)}+\frac{l^{2} A}{2(1+\mu) I}$

is called as the size effect parameter of the AFG microbeam because it is related to the material length-scale parameter 1. For an AFG micro-beam with rectangular cross-section, we express the size effect parameter as

$\alpha=\frac{1-\mu}{(1+\mu)(1-2 \mu)}+\left(\frac{l}{h}\right)^{2} \frac{6}{(1+\mu)}$

In order to express the size effect of the bending stiffness of the AFG micro-beam, we define a dimensionless bending stiffness as

$K^{\prime}(x)=\frac{K(x)}{E_{0} I}$

Inserting Eq. (14) into Eq. (18), we formulate the dimensionless bending stiffness of the AFG micro-beam as $K^{\prime}(x)=\alpha\left[1+(e-1) \sin \frac{\pi x}{L}\right]$

where

$e=\frac{E_{1}}{E_{0}}$

is called as the FG parameter of the AFG micro-beam.

\subsection{Deflection formulation}

According to the displacement boundary conditions of the AFG micro-beam in Figure 1, we assume the deflection formulation as

$w(x)=C_{1} \sin \left(\frac{\pi x}{L}\right)+C_{2} \sin \left(\frac{3 \pi x}{L}\right)$

where $C_{1}$ and $C_{2}$ are the undetermined coefficients. The total potential energy of the AFG micro-beam reads as $\Pi=U+V$

where $\mathrm{U}$ is the strain energy formulated by Eq. (13), and

$V=-\int_{0}^{L} q \cdot w(x) d x$

is the loaded potential energy.

Plugging the strain energy Eq. (13), loaded potential energy Eq. (23) and deflection formulation Eq. (21) into the total potential energy Eq. (22), we express the total potential energy of the AFG micro-beam in Figure 1 as

$$
\begin{array}{r}
\Pi\left(C_{1}, \mathrm{C}_{2}\right)=\frac{\alpha E_{0} I \pi^{3}}{L^{3}}\left\{\left[\frac{\pi}{4}+\frac{2(e-1)}{3}\right] C_{1}^{2}-\frac{12(e-1)}{5} C_{1} C_{2}+\left[\frac{81 \pi}{4}+\frac{1458(e-1)}{35}\right] C_{2}^{2}\right\} \\
-q C_{1} \frac{2 L}{\pi}-q C_{2} \frac{2 L}{3 \pi}
\end{array}
$$

According to the principle of minimum total potential 
energy, the actual displacement field minimizes the total potential energy of an elastomer. Hence, the first order variation of the total potential energy expressed by Eq. (24) should be zero, i.e.

$\delta \Pi\left(C_{1}, C_{2}\right)=\frac{\partial \Pi}{\partial C_{1}} \delta C_{1}+\frac{\partial \Pi}{\partial C_{2}} \delta C_{2}=0$

Eq. (25) should be identical for arbitrary $\delta C_{1}$ and $\delta C_{2}$, which asks for

$$
\begin{aligned}
& \frac{\partial \Pi}{\partial C_{1}}=0 \\
& \frac{\partial \Pi}{\partial C_{2}}=0
\end{aligned}
$$

Substituting the expression of total potential energy Eq. (24) into Eqs. (26a) and (26b), we obtain an algebraic equation set. Solving the obtained algebraic equation set, we have

$C_{1}=\frac{2 q L^{4}}{3 \pi^{4} E_{0} I \alpha} \cdot \frac{a_{2}+3 a_{3}}{a_{1} a_{3}-a_{2}^{2}}$

$C_{2}=\frac{2 q L^{4}}{3 \pi^{4} E_{0} I \alpha} \cdot \frac{a_{1}+3 a_{2}}{a_{1} a_{3}-a_{2}^{2}}$

$$
\text { where }
$$

$a_{1}=\frac{3 \pi+8(e-1)}{6}$

$a_{2}=\frac{12(e-1)}{5}$

$a_{3}=\frac{2835 \pi+5832(e-1)}{70}$

Substituting Eq. (27) into the deflection formulation Eq. (21), we obtain

$$
w(x)=\frac{2 q L^{4}}{3 \pi^{4} E_{0} I \alpha} \cdot\left[\frac{a_{2}+3 a_{3}}{a_{1} a_{3}-a_{2}^{2}} \cdot \sin \frac{\pi x}{L}+\frac{a_{1}+3 a_{2}}{a_{1} a_{3}-a_{2}^{2}} \cdot \sin \frac{3 \pi x}{L}\right]
$$

In order to express the size effect of the deflection of an AFG micro-beam, we define a dimensionless deflection as

$$
w^{\prime}(x)=w(x) /\left(\frac{2 q L^{4}}{3 \pi^{4} E_{0} I}\right)
$$

Substituting Eq. (29) into Eq. (30),we formulate the dimensionless deflection of the AFG micro-beam in Figure 1 as

$$
w^{\prime}(x)=\frac{1}{\alpha} \cdot\left[\frac{a_{2}+3 a_{3}}{a_{1} a_{3}-a_{2}^{2}} \cdot \sin \left(\frac{\pi x}{L}\right)+\frac{a_{1}+3 a_{2}}{a_{1} a_{3}-a_{2}^{2}} \cdot \sin \left(\frac{3 \pi x}{L}\right)\right]
$$

\subsection{Normal stress formulation}

Plugging the deflection formulation Eq. (29) into the stress expression Eq. (10), we formulate the normal stress in a generic matter point of the AFG micro-beam cross-section as

$$
\begin{aligned}
& \sigma_{x x}=\frac{2 q L^{2} z}{3 \pi^{2} I} \cdot \frac{E^{\prime}(x)}{\alpha} \cdot\left(\frac{a_{2}+3 a_{3}}{a_{1} a_{3}-a_{2}^{2}} \cdot \sin \frac{\pi x}{L}+\frac{9 a_{1}+27 a_{2}}{a_{1} a_{3}-a_{2}^{2}} \cdot \sin \frac{3 \pi x}{L}\right) \\
& \quad \text { where } \\
& E^{\prime}(x)=\frac{1-\mu}{(1+\mu)(1-2 \mu)}\left[1+(e-1) \sin \frac{\pi x}{L}\right]
\end{aligned}
$$

In order to analyze the size effect of the normal stress of an AFG micro-beam, we define a dimensionless normal stress as

$\sigma_{x x}^{\prime}=\sigma_{x x} /\left(\frac{2 q L^{2} z_{\max }}{3 \pi^{2} I}\right)$

Substituting Eq. (32) into Eq. (34), we formulate the dimensionless normal stress of the AFG micro-beam in Figure 1 as

$\sigma_{x x}^{\prime}=\frac{E^{\prime}(x)}{\alpha} \cdot\left(\frac{a_{2}+3 a_{3}}{a_{1} a_{3}-a_{2}^{2}} \cdot \sin \frac{\pi x}{L}+\frac{9 a_{1}+27 a_{2}}{a_{1} a_{3}-a_{2}^{2}} \cdot \sin \frac{3 \pi x}{L}\right) \cdot \frac{z}{z_{m z x}}$

\subsection{Couple stress formulation}

Plugging the deflection formulation Eq. (29) into the couple stress expression Eq. (11), we formulate the couple stress in a generic point of the AFG micro-beam cross-section as

$m_{x y}=\frac{2 q L^{2} h^{2}}{3 \pi^{2} I} \cdot \frac{G^{\prime}(x)}{\alpha} \cdot\left(\frac{l}{h}\right)^{2} \cdot\left[\frac{a_{2}+3 a_{3}}{a_{1} a_{3}-a_{2}^{2}} \cdot \sin \frac{\pi x}{L}+\frac{9 a_{1}+27 a_{2}}{a_{1} a_{3}-a_{2}^{2}} \cdot \sin \frac{3 \pi x}{L}\right]$

where

$G^{\prime}(x)=\frac{1}{2(1+\mu)}\left[1+(e-1) \sin \frac{\pi x}{L}\right]$

In order to analyze the size effect of the couple stress of the AFG micro-beam, we define a dimensionless couple stress as

$m_{x y}^{\prime}=m_{x y} /\left(\frac{2 q L^{2} h^{2}}{3 \pi^{2} I}\right)$

Substituting Eq. (36) into Eq. (38), we formulate the dimensionless couple stress of the AFG micro-beam as

$m_{x y}^{\prime}=\frac{G^{\prime}(x)}{\alpha} \cdot\left(\frac{l}{h}\right)^{2} \cdot\left[\frac{a_{2}+3 a_{3}}{a_{1} a_{3}-a_{2}^{2}} \cdot \sin \frac{\pi x}{L}+\frac{9 a_{1}+27 a_{2}}{a_{1} a_{3}-a_{2}^{2}} \cdot \sin \frac{3 \pi x}{L}\right]$

\section{Size effects of AFG micro-beam}

\subsection{Size effect of bending stiffness}

The size effect of bending stiffness of the AFG micro-beam in Figure 1 are numerically investigated in this section. Figure 2 plots the bending stiffness curves related to size effect, where the dimensionless bending stiffness is calculated by Eq. (19) and maximum dimensionless bending stiffness is specified by Eq. (19) with $x=L / 2$, respectively.

Figure 2(a) plots the dimensionless bending stiffness curves versus dimensionless coordinate with respect to different values of dimensionless height. It is clear that each dimensionless bending stiffness curve of the AFG microbeam forms a sinusoidal hump whose peak declines with the increased value of dimensionless height. This indicates the size effect of bending stiffness that the smaller the value of dimensionless height is, the larger the value of dimensionless bending stiffness of the AFG micro-beam is.

Figure 2(b) shows the maximum dimensionless bending stiffness curves versus dimensionless height with respect to different values of FG parameter. It is found that the value of maximum dimensionless bending stiffness rapidly decreases with the increased value of dimensionless height when the dimensionless height is less than 4, however it gradually becomes a stable constant when the value of dimensionless height is greater than 10 . This explains the size effect of bending stiffness that it is obvious when the value of dimensionless height is less than 4 , while it can be neglected when the value of dimensionless height 
is greater than 10. It is seen that the curve of maximum dimensionless bending stiffness moves upward and extends vertically when the FG parameter becomes larger. This explains the influence of FG parameter on the size effect of bending stiffness that the larger the value of FG parameter is, the more obvious the size effect of bending stiffness is.
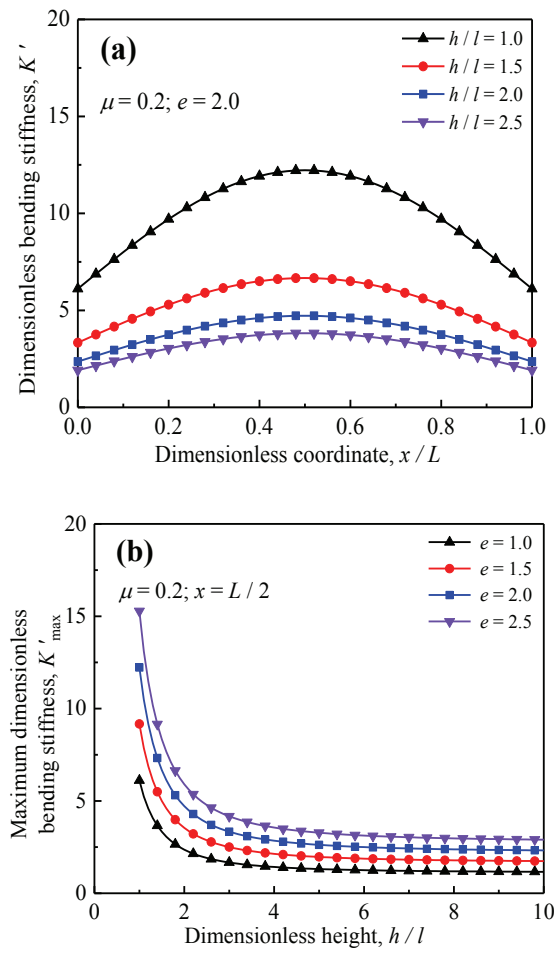

Figure 2 Bending stiffness curves related to the size effect: (a) dimensionless bending stiffness vs. dimensionless coordinate with different value of dimensionless height, and (b) maximum dimensionless bending stiffness vs. dimensionless height with different value of FG parameter.

\subsection{Size effect of deflection}

The size effect of deflection of the AFG micro-beam in Figure 1 is numerically investigated in this section. Figure 3 plots the deflection curves related to the size effect, where the dimensionless deflection is calculated by Eq. (31) and maximum dimensionless deflection is specified by Eq. (31) with $\mathrm{x}=\mathrm{L} / 2$, respectively.

Figure 3 (a) plots the dimensionless deflection curves versus dimensionless coordinate with respect to different values of dimensionless height. It is clear that each dimensionless deflection curve of the AFG micro-beam forms a different sinusoidal hump whose peak rises with the increased dimensionless height. This indicates the size effect of deflection that the bending flexibility of an AFG micro-beam rises with the increased value of dimensionless height.

Figure 3 (b) shows the maximum dimensionless deflection curves versus dimensionless height with respect to different values of FG parameter. It is found that the value of maximum dimensionless deflection obviously increases with the increased value of dimensionless height when the value of dimensionless height is less than 4 , however it gradually becomes a stable constant when the value of dimensionless height is greater than 10. This illustrates the size effect of deflection that it is obvious when the value of dimensionless height is less than 4 , however it can be neglected when the value of dimensionless height is greater than 10. It is clear that the curve of maximum dimensionless deflection moves downward and shrinks vertically when the FG parameter becomes larger. This explains the influence of FG parameter on the size effect of deflection that the smaller the value of FG parameter is, the more obvious the size effect of deflection of an AFG micro-beam is.
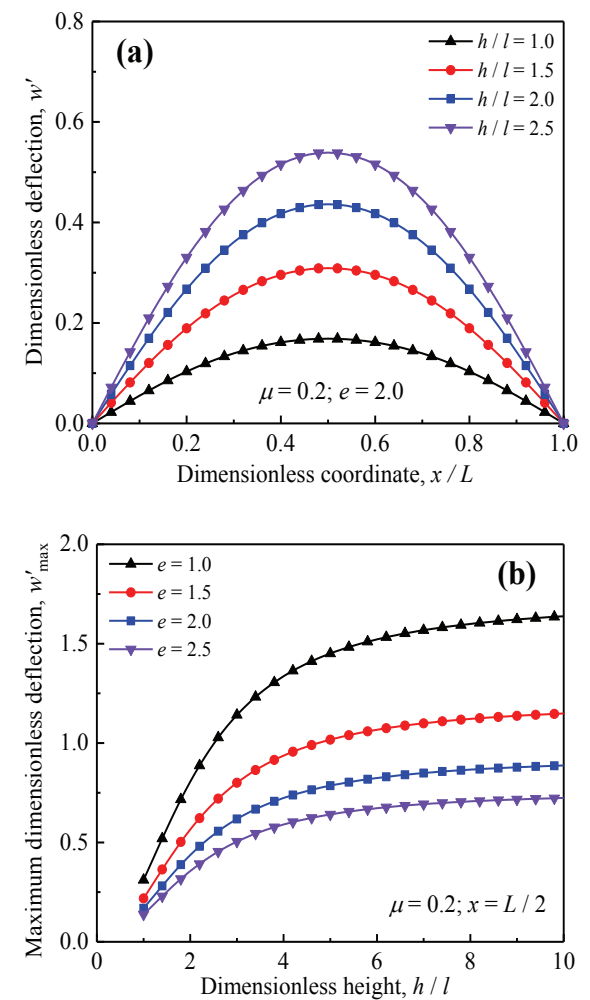

Figure 3 Deflection curves related to the size effect: (a) dimensionless deflection vs. dimensionless coordinate with different value of dimensionless height, and (b) maximum dimensionless deflection vs. dimensionless height with different value of FG parameter.

\subsection{Size effect of normal stress}

The size effect of normal stress of the AFG micro-beam in Figure 1 is numerically investigated in this section. Figure 4 plots the normal stress curves related to the size effect, where the dimensionless normal stress is calculated by Eq. (35) with $\mathrm{z}=\mathrm{h} / 2$ and maximum dimensionless normal stress is specified by Eq. (35) with $z=h / 2$ and $x=L / 2$, respectively.

Figure 4(a) shows the dimensionless normal stress curves versus dimensionless coordinate with respect to different values of dimensionless height. It is clear that each dimensionless normal stress curve is with a platform 
which is due to the FG effect of an AFG micro-beam. The altitude of platform of dimensionless normal stress rises with the increased value of dimensionless height, which explains the size effect of normal stress that the larger the value of dimensionless height is, the larger the value of dimensionless normal stress is.

Figure 4(b) plots the maximum dimensionless normal stress curves versus dimensionless height with respect to different values of FG parameter. It is found that the value of maximum dimensionless normal stress nonlinearly increases with the increased value of dimensionless height when the value of dimensionless height is below 10, however it gradually becomes a stable constant when the value of dimensionless height is above 20. This indicates that the size effect of normal stress of an AFG microbeam is obvious when the value of dimensionless height is below 10, however it can be neglected when the value of dimensionless height is above 20. It is seen that the influence of FG parameter on the size effect of normal stress is not very obvious.
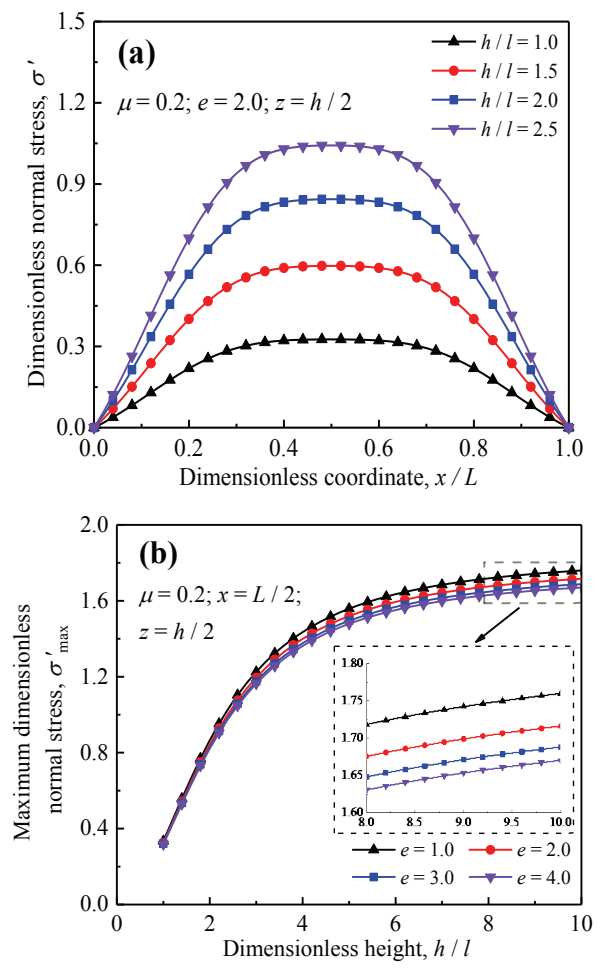

Figure 4 Normal stress curves related to the size effect: (a) dimensionless normal stress vs. dimensionless coordinate with different value of dimensionless height, and (b) the maximum dimensionless normal stress vs. dimensionless height with different value of FG parameter.

\subsection{Size effect of couple stress}

The size effect of couple stress of the AFG micro-beam in Figure 1 is numerically investigated in this section. Figure 5 plots the couple stress curves related to the size effect, where the dimensionless couple stress is calculated by Eq. (39) and maximum dimensionless couple stress is specified by Eq. (39) with $x=L / 2$, respectively.
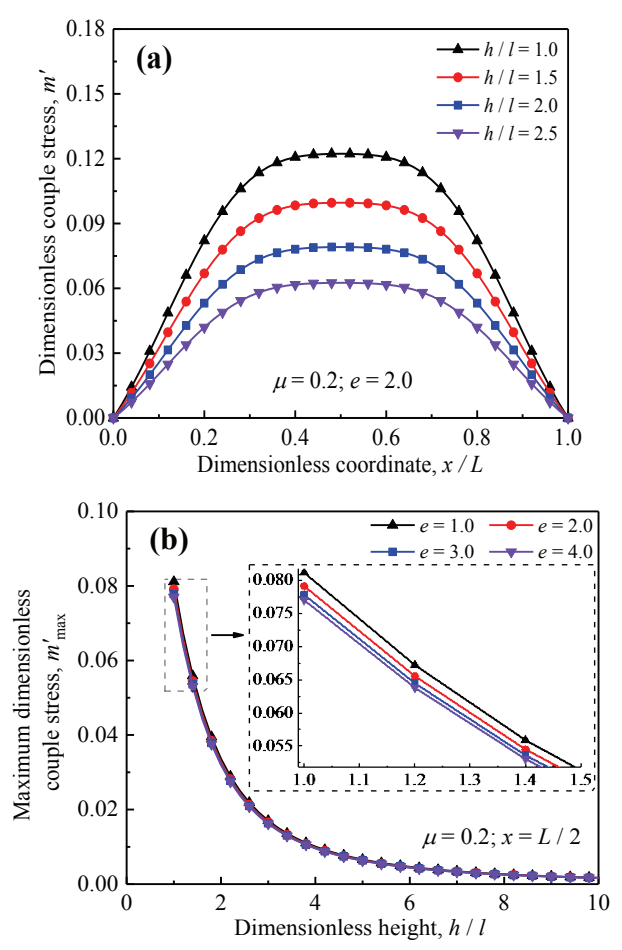

Figure 5 Couple stress curves related to the size effect: (a) dimensionless couple stress vs. dimensionless coordinate with different value of dimensionless height, and (b) maximum dimensionless couple stress vs. dimensionless height with different value of FG parameter.

Figure 5(a) shows the dimensionless couple stress curves versus dimensionless coordinate with different values of dimensionless height. It is also clear that each dimensionless couple stress curve is with a platform due to the FG effect of an AFG micro-beam. But the altitude of platform of dimensionless normal stress declines with the increased value of dimensionless height, which indicates the size effect of couple stress of an AFG micro-beam that the smaller the value of dimensionless height is, the larger the value of dimensionless couple stress is.

Figure 5(b) shows the maximum dimensionless couple stress curves versus dimensionless height with different values of FG parameter. It is seen that the value of maximum dimensionless couple stress rapidly decreases with the increased value of dimensionless height when the value of dimensionless height is below 5, however it gradually becomes a stable constant when the value of dimensionless height is above 10. This indicates the size effect of couple stress of an AFG micro-beam that it is obvious when the value of dimensionless height is below 5 , while it can be neglected when the value of dimensionless height is above 10. It is also seen that the influences of FG parameter on the size effect of couple stress of an AFG micro-beam is not obvious.

\section{FG effects of AFG micro-beam}

\subsection{FG effect of bending stiffness}

The FG effect of bending stiffness of the AFG micro-beam in 
Figure 1 is numerically investigated in this section. Figure 6 shows the bending stiffness curves related to the FG effect, where the dimensionless bending stiffness is calculated by Eq. (19) and maximum dimensionless bending stiffness is specified by Eq. (19) with $\mathrm{x}=\mathrm{L} / 2$, respectively.
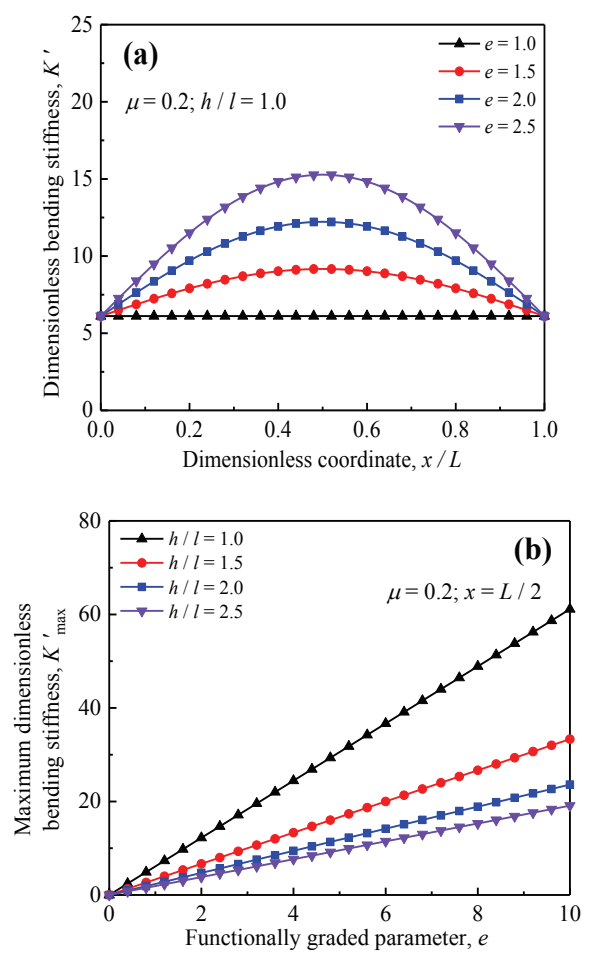

Figure 6 Bending stiffness curves related to the FG effect: (a) dimensionless bending stiffness vs. dimensionless coordinate with different value of FG parameter, and (b) dimensionless bending stiffness vs. FG parameter with different value of dimensionless height.

Figure 6(a) plots the dimensionless bending stiffness curves versus dimensionless coordinate with respect to different values of FG parameter. It is clear that the peak of dimensionless bending stiffness curve rises with the increased value of FG parameter, which is due to the expression of elastic modulus Eq. (5), and the definition of FG parameter Eq. (20). This illustrates the FG effect of bending stiffness of an AFG micro-beam that the bending stiffness increases with the increased value of FG parameter.

Figure 6(b) plots the maximum dimensionless bending stiffness curves versus FG parameter with respect to different values of dimensionless height. It is clear that the value of maximum dimensionless bending stiffness increases with the increased value of FG parameter, which also indicates FG effect of bending stiffness of an AFG micro-beam. It is found that the curve of maximum dimensionless bending stiffness extends vertically with the decreased value of dimensionless height, which indicates the influence of dimensionless height on the FG effect of bending stiffness that the smaller the value of dimensionless height is, the more obvious the FG effect of an AFG microbeam is.

\subsection{FG effect of deflection}

The FG effect of deflection of the AFG micro-beam in Figure 1 is numerically investigated in this section. Figure 7 shows the deflection curves related to the FG effect, where the dimensionless deflection is calculated by Eq. (31) and maximum dimensionless deflection is specified by Eq. (31) with $\mathrm{x}=\mathrm{L} / 2$, respectively.
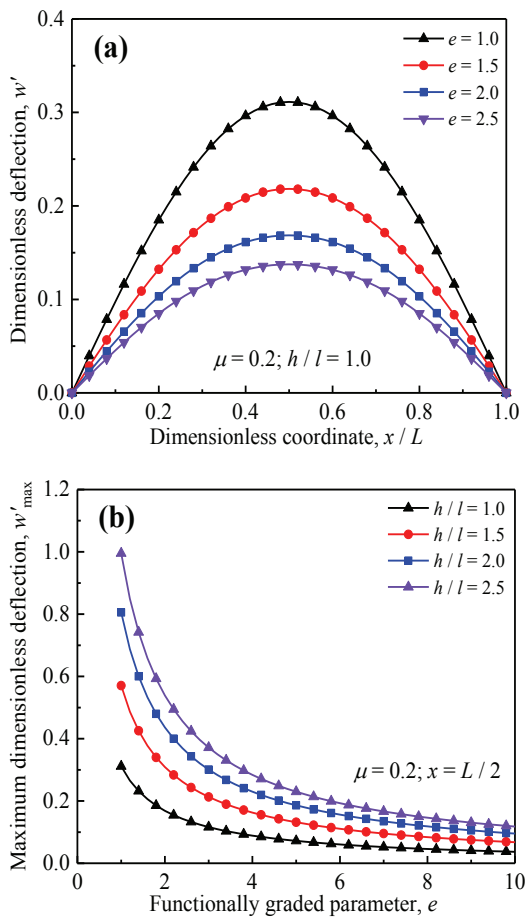

Figure 7 Deflection curves related to the FG effect of deflection: (a) dimensionless deflection vs. dimensionless coordinate with different value of FG parameter, and (b) maximum dimensionless deflection vs. FG parameter with different value of dimensionless height.

Figure 7(a) shows the dimensionless deflection curves versus dimensionless coordinate with respect to different values of FG parameter. It is clear that the peak of sinusoidal curve of dimensionless deflection decreases with the increased value of FG parameter. This illustrates the FG effect of deflection that the bending flexibility of an AFG micro-beam decreases with the increased value of FG parameter.

Figure 7(b) plots the maximum dimensionless deflection curves versus FG parameter with respect to different values of dimensionless height. It is found that the value of maximum dimensionless deflection rapidly decreases with the increased value of FG parameter when the FG parameter is below 5, however it gradually becomes a stable constant when the FG parameter is above 10 . This means that the FG effect of deflection of an AFG microbeam is obvious when the FG parameter is below 5, while it can be neglected when the FG parameter is above 10 It is clear that the maximum dimensionless deflection curve moves upward and extends vertically when the dimensionless height becomes larger. This explains the 
influence of dimensionless height on the FG effect of deflection of an AFG micro-beam that the larger the value of dimensionless height is, the more obvious the FG effect is.

\subsection{FG effect of normal stress}

The FG effect of normal stress of the AFG micro-beam in Figure 1 is numerically investigated in this section. Figure 8 plots the normal stress curves related to the FG effect, where the dimensionless normal stress is calculated by Eq. (35) with $\mathrm{z}=\mathrm{h} / 2$ and maximum dimensionless normal stress is specified by Eq. (35) with $z=h / 2$ and $x=L / 2$, respectively.
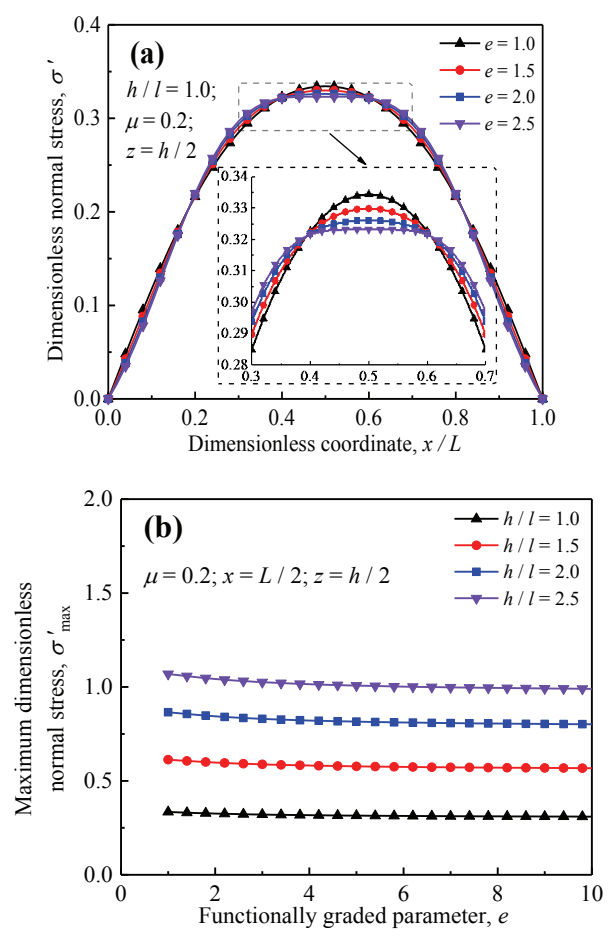

Figure 8 Normal stress curves related to the FG effect: (a) dimensionless normal stress vs. dimensionless coordinate with different value of FG parameter, and (b) maximum dimensionless normal stress vs. FG parameter with different value of dimensionless height.

Figure 8(a) shows the dimensionless normal stress curves versus dimensionless coordinate with respect to different values of FG parameter. It is found that the curve peak of dimensionless normal stress decreases with the increased value of FG parameter. However the curves of dimensionless normal stress for different FG parameters are very close and similar, which indicates that the FG effect of normal stress of an AFG micro-beam is not obvious.

Figure 8(b) plots the maximum dimensionless normal stress curves versus FG parameter with respect to different values of dimensionless height. It is clear that the value of maximum dimensionless normal stress has only slight decrease with the increased value of FG parameter, which also means that the FG effect of normal stress of an AFG micro-beam is not obvious. It is seen that the maximum dimensionless normal stress curve moves upward and becomes more declining with the increased value of dimensionless height. This explains that the larger the value of dimensionless height is, the more obvious the FG effect of normal stress of an AFG micro-beam is.

\subsection{FG effect of couple stress}

The FG effect of couple stress of the AFG micro-beam in Figure 1 is numerically investigated in this section. Figure 9 plots the couple stress curves related to the FG effect, where the dimensionless couple stress is calculated by Eq. (39) and maximum dimensionless couple stress is specified by Eq. (39) with $x=L / 2$, respectively.
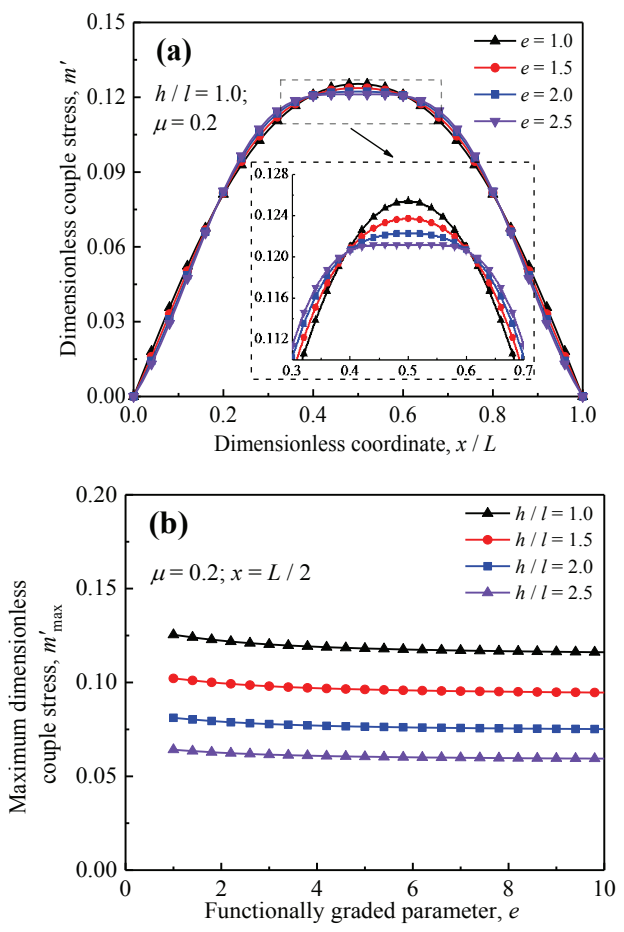

Figure 9 Couple stress curves related to the FG effect: (a) dimensionless couple stress vs. dimensionless coordinate with different value of FG parameter, and (b) maximum dimensionless couple stress vs. FG parameter with different value of dimensionless height.

Figure 9(a) plots the dimensionless couple stress curves versus dimensionless coordinate with respect to the different values of FG parameter. It is found that the curve peak of dimensionless couple stress decreases with the increased value of FG parameter. However the curves of dimensionless couple stress for the different values of FG parameter are very close and similar, which indicates that the FG effect of couple stress of an AFG micro-beam is also not obvious.

Figure 9(b) shows the maximum dimensionless couple stress curves versus FG parameter with respect to different values of dimensionless height. It is found that the value of maximum dimensionless couple stress has only slight decrease with the increased value of FG parameter, which also means that the FG effect of couple stress of an AFG micro-beam is not obvious. It is clear that the maximum dimensionless couple stress curve moves upward and becomes more declining with the decreased 
dimensionless height. This explains that the smaller the value of dimensionless height is, the more obvious the FG effect of couple stress of an AFG micro-beam is.

\section{Poisson effects of AFG micro-beam}

\subsection{Poisson effect of bending stiffness}

The Poisson effect of bending stiffness of the AFG microbeam in Figure 1 is numerically investigated in this section. Figure 10 shows the bending stiffness curves related to the Poisson effect, where the dimensionless bending stiffness is calculated by Eq. (19) and maximum dimensionless bending stiffness is specified by Eq. (19) with $x=h / 2$, respectively.
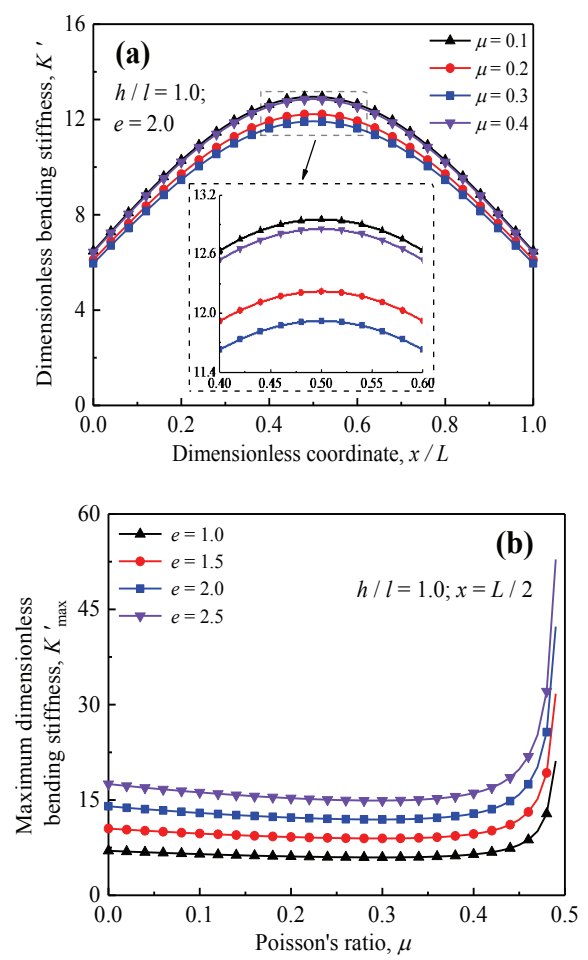

Figure 10 Bending stiffness curves related to the Poisson effect: (a) dimensionless bending stiffness vs. dimensionless coordinate with different value of Poisson's ratio, and

(b) maximum dimensionless bending stiffness vs. Poisson's ratio with different value of FG parameter.

Figure 10(a) plots the dimensionless bending stiffness curves versus dimensionless coordinate with respect to different values of Poisson's ratio. It is clear that each dimensionless bending stiffness curve is with a sinusoidal shape. The Poisson's ratio has an obvious influence on the curve of dimensionless bending stiffness of an AFG microbeam, which is the Poisson effect of bending stiffness.

Figure 10(b) shows the maximum dimensionless bending stiffness curves versus Poisson's ratio with respect to different values of FG parameter. It is seen that the value of maximum dimensionless bending stiffness slightly decreases with the increased value of Poisson's ratio when the value of Poisson's ration is below 0.4 , however it rapidly increases with the increased value of Poisson's ratio when the value of Poisson's ration is above 0.4. This indicates that the Poisson effect of bending stiffness is obvious when the value of Poisson's ration is above 0.4 , while it can be neglected when the value of Poisson's ration is below 0.4. It is seen that the maximum dimensionless bending stiffness curve moves upward and extends vertically when the value of FG parameter becomes larger. This explains the influence of FG parameter on the Poisson effect of bending stiffness that the larger the value of FG parameter is, the more obvious the Poisson effect of bending stiffness of an AFG micro-beam is.

\subsection{Poisson effect of deflection}

The Poisson effect of deflection of the AFG micro-beam in Figure 1 is numerically investigated in this section. Figure 11 shows the deflection curves related to the Poisson effect, where the dimensionless deflection is calculated by Eq. (31) and maximum dimensionless deflection is specified by Eq. (31) with $\mathrm{x}=\mathrm{L} / 2$, respectively.
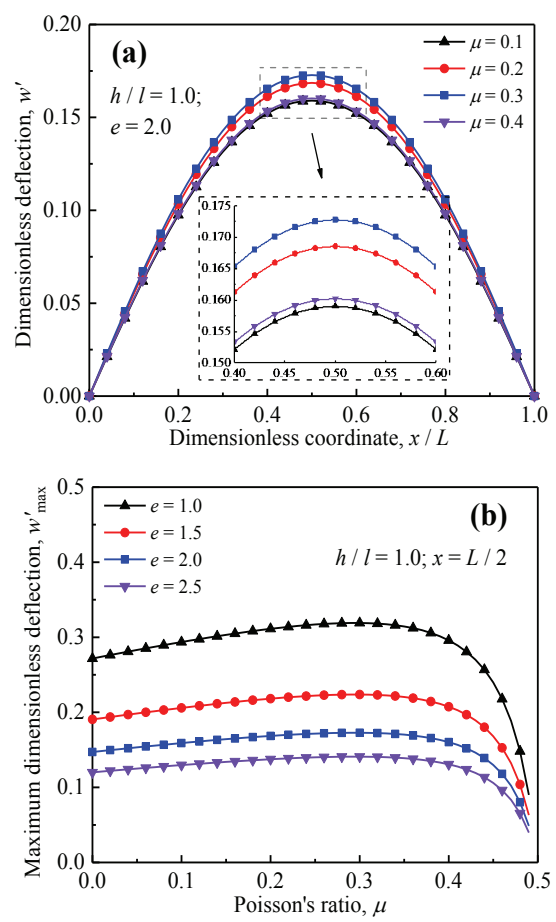

Figure 11 Deflection curves related to the Poisson effect: (a) dimensionless deflection vs. dimensionless coordinate with different value of Poisson's ratio, and (b) maximum dimensionless deflection vs. Poisson's ratio with different value of FG parameter.

Figure 11(a) plots the dimensionless deflection curves versus dimensionless coordinate with respect to different values of Poisson's ratio. It is found that each dimensionless deflection curve is with a sinusoidal shape and the Poisson's ratio has an obvious influence on the curve of dimensionless deflection, which is the Poisson effect of deflection of an AFG micro-beam.

Figure 11(b) shows the maximum dimensionless deflection curves versus Poisson's ratio with respect to different values of FG parameter. It is clear that the value 
of maximum dimensionless deflection slightly increases with the increased value of Poisson's ratio when the value of Poisson's ratio is below 0.4 , however it rapidly decreases with the increased value of Poisson's ratio when the value of Poisson's ratio is above 0.4 . This indicates that the Poisson effect of deflection of an AFG micro-beam is obvious when the value of Poisson's ratio is above 0.4 , while it can be neglected when the value of Poisson's ratio is below 0.4. It is seen that the maximum dimensionless deflection curve moves downward and shrinks vertically with the increased value of FG parameter. This explains the influence of FG parameter on the Poisson effect of deflection that the smaller the value of FG parameter is, the more obvious the Poisson effect of deflection of an AFG micro-beam is.

\subsection{Poisson effect of normal stress}

The Poisson effect of normal stress of the AFG micro-beam in Figure 1 is numerically investigated in this section. Figure 12 shows the normal stress curves related to the Poisson effect, where the dimensionless normal stress is calculated by Eq. (35) with $\mathrm{z}=\mathrm{h} / 2$ and the maximum dimensionless normal stress is specified by Eq. (35) with $\mathrm{z}=\mathrm{h} / 2$ and $\mathrm{x}=$ $\mathrm{L} / 2$, respectively.
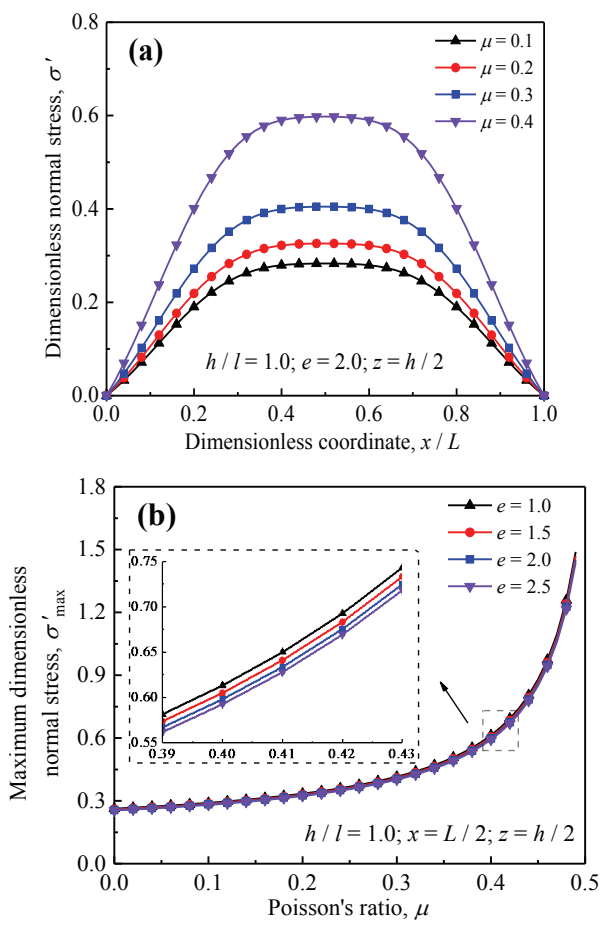

Figure 12 Normal stress curves related to the Poisson effect: (a) dimensionless normal stress vs. dimensionless coordinate with different value of Poisson's ratio, and (b) maximum dimensionless normal stress vs. Poisson's ratio with different value of FG parameter.

Figure 12(a) shows the dimensionless normal stress curves versus dimensionless coordinate with respect to different values of Poisson's ratio. It is clear that a platform appears in the dimensionless normal stress curve due to the FG effect of an AFG micro-beam. The altitude of platform of dimensionless normal stress increases with the increased value of Poisson's ratio. This indicates the influence of Poisson's ratio on the dimensionless normal stress, which is the Poisson effect of normal stress of an AFG micro-beam.

Figure 12(b) shows the maximum dimensionless normal stress curves versus Poisson's ratio with respect to different values of FG parameter. It is seen that the value of maximum dimensionless normal stress nonlinearly increases with the increased value of Poisson's ratio, which means that the Poisson effect of normal stress of an AFG micro-beam is obvious. It is found that the maximum dimensionless normal stress curves with different values of FG parameter are very close and similar, which means that the influence of FG parameter on the Poisson effect of normal stress of an AFG micro-beam is not obvious and then can be neglected.

\subsection{Poisson effect of couple stress}

The Poisson effect of couple stress of the AFG micro-beam in Figure 1 is numerically investigated in this section. Figure 13 shows the couple stress curves related to the Poisson effect, where the dimensionless couple stress is calculated by Eq. (39) and maximum dimensionless couple stress is specified by Eq. (39) with $\mathrm{x}=\mathrm{L} / 2$, respectively.
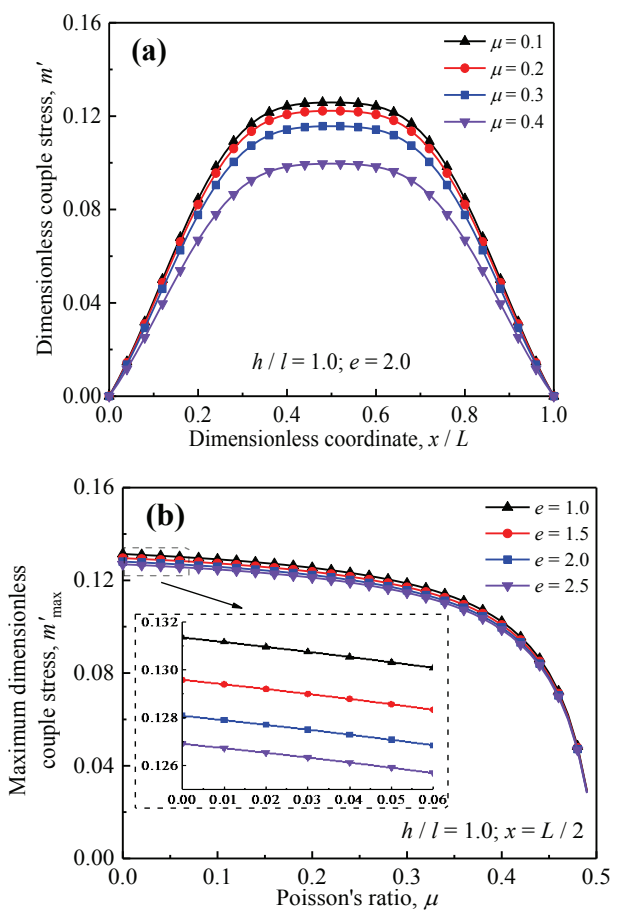

Figure 13 Couple stress curves related to the Poisson effect: (a) dimensionless couple stress vs. dimensionless coordinate with different value of Poisson's ratio, and (b) maximum dimensionless couple stress vs. Poisson's ratio with different value of FG parameter.

Figure 13(a) shows the dimensionless couple stress curves versus dimensionless coordinate with respect to different values of Poisson's ratio. It is clear that a platform also appears in the dimensionless couple stress curve due to the FG effect of couple stress of an AFG micro-beam. The altitude of platform decreases with the increased value of 
Poisson's ratio. This explains the influence of Poisson's ratio on the dimensionless couple stress, which is the Poisson effect of couple stress of an AFG micro-beam.

Figure 13(b) plots the maximum dimensionless couple stress curves versus Poisson's ratio with respect to different values of FG parameter. It is seen that the value of maximum dimensionless couple stress nonlinearly decreases with the increased value of Poisson's ratio, which indicates that the Poisson effect of couple stress of an AFG micro-beam is obvious. It is found that maximum dimensionless couple stress curves for different values of FG parameter are very close and similar, which indicates that the influence of FG parameter on the Poisson effect of couple stress of an AFG micro-beam is not obvious and then can be neglected.

\section{Conclusions}

The components of strain, curvature, stress and couple stress of an AFG micro-beam are described according to the MCST and Bernoulli-Euler theory. The size-dependent model related to FG effect and Poisson effect is developed to describe and predict the mechanical behaviors of the AFG micro-beam by using the principle of minimum potential energy. The mechanical behaviors of the AFG micro-beam, which are related to size the effects, FG effects and Poisson effects, are numerically investigated via the dimensionless definitions such as dimensionless bending stiffness, dimensionless deflection, dimensionless normal stress and dimensionless couple stress. Through the numerical simulation, some important conclusions are summarized as follows.

1) The size effects of mechanical behaviors, which includes the size effects of bending stiffness, deflection, normal stress and couple stress of the AFG micro-beam, are obvious when the value of dimensionless height is small enough. However the size effects can be neglected when the value of dimensionless height of micro-beam is large enough.

2) The FG effects, i.e. the influences of FG parameter, are important factors related to the bending stiffness and deflection of AFG micro-beam. However the FG effects of normal stress and couple stress are not very obvious. The dimensionless height has visible influences on the FG effects of bending stiffness, deflection, normal stress and couple stress of the AFG micro-beam.

3) The Poisson effects, i.e. the influences of Poisson's ratio, are not negligent for the bending stiffness, deflection, normal stress and couple stress of the AFG micro-beam. The dimensionless height has obvious influences only on the Poisson effects of bending stiffness and deflection, while it has little influences on the Poisson effects of normal stress and couple stress.

Author Contributions: Bo ZHOU first proposed a method to analyze the size effect, functionally graded (FG) effect and Poisson effect of an axially functionally graded (AFG) micro-beam by using Bernoulli-Euler beam theory and modified coupled stress theory and combining with the principle of minimum potential energy. Shuai WANG established a size-dependent model related to FG effect and Poisson effect, including the calculation formulas of bending stiffness, deflection, normal stress and couple stress, and numerically investigated the size effect, FG effect and Poisson effect of simply supported AFG micro-beam of the established model. Zetian KANG and Shichen ZHOU determined the displacement field of AFG micro-beam based on Bernoulli-Euler beam theory. And expressed the components of strain, curvature, stress and couple stress according to the modified coupling stress theory. Using the principle of minimum potential energy. Shifeng XUE put forward some guiding opinions on the logicality and innovation of the article.

Conflict of Interest: We are committed to that there is no conflict of interest regarding the publication of this paper.

Acknowledgments: The authors of this paper acknowledge the supports from the National Key Research and Development Program of China (Grant No. 2017YFC0307604) and the Talent Foundation of China University of Petroleum (Grant No. Y1215042).

This is the Appendix: This article does not cover the details that require an appendix.

\section{References}

[1] Asghari M, Ahmadian M T, Kahrobaiyan M H, Rahaeifard M, 2010. On the size-dependent behavior of functionally graded micro-beams. Materials \& Design. 31(5), 23242329. https://doi.org/10.1016/j.matdes.2009.12.006

[2] Dai H L, Wang Y K, Wang L, 2015. Nonlinear dynamics of cantilevered microbeams based on modified couple stress theory. International Journal of Engineering Science. 94, 103-112. https://doi.org/10.1016/j. ijengsci.2015.05.007

[3] Dehrouyeh-Semnani A M, Nikkhah-Bahrami M, 2015a. The influence of size-dependent shear deformation on mechanical behavior of microstructures-dependent beam based on modified couple stress theory. Composite Structures. 123, 325-336. https://doi.org/10.1016/j. compstruct.2014.12.038

[4] Dehrouyeh-Semnani A M, Nikkhah-Bahrami M, 2015b. A discussion on incorporating the poisson effect in microbeam models based on modified couple stress theory. International Journal of Engineering Science. 86, 20-25. https://doi.org/10.1016/j.ijengsci.2014.10.003

[5] Ghayesh M H, Farokhi H, Gholipour A, Tavallaeinejad M, 2017. Nonlinear bending and forced vibrations of axially functionally graded tapered microbeams. International Journal of Engineering Science. 120, 51-62. https://doi. org/10.1016/j.ijengsci.2017.03.010

[6] Heydari A, Jalali A, Nemati A, 2017. Buckling analysis of circular functionally graded plate under uniform radial compression including shear deformation with linear 
and quadratic thickness variation on the Pasternak elastic foundation. Applied Mathematical Modelling. 41, 494-507. https://doi.org/10.1016/j.apm.2016.09.012

[7] Huang Y, Li X F, 2010. A new approach for free vibration of axially functionally graded beams with non-uniform cross-section. Journal of Sound and Vibration. 329(11), 2291-2303. https://doi.org/10.1016/j.jsv.2009.12.029

[8] Karamanlı A, Vo T P, 2018. Size dependent bending analysis of two directional functionally graded microbeams via a quasi-3D theory and finite element method. Composites Part B: Engineering. 144, 171-183. https://doi.org/10.1016/j.compositesb.2018.02.030

[9] Ke L L, Wang Y S, 2011. Size effect on dynamic stability of functionally graded micro-beams based on a modified couple stress theory. Composite Structure. 93(2), 342350. https://doi.org/10.1016/j.compstruct.2010.09.008

[10] Ke L L, Wang Y S, Yang J, Kitipornchai S, 2012a. Free vibration of size-dependent Mindlin microplates based on the modified couple stress theory. Journal of Sound and Vibration. 331(1), 94-106. https://doi.org/10.1016/j. jsv.2011.08.020

[11] Ke L L, Yang J, Kitipornchai S, Bradford M A, 2012b. Bending, buckling and vibration of size-dependent functionally graded annular microplates. Composite Structures. 94(11), 3250-3257. https://doi.org/10.1016/j. compstruct.2012.04.037

[12] Kong S L, Zhou S J, Nie Z F, Wang K, 2008. The sizedependent natural frequency of Bernoulli-Euler micro-beams. International Journal of Engineering Science. 46(5), 427-437. https://doi.org/10.1016/j. ijengsci.2007.10.002

[13] Kouzeli M, Mortensen A, 2002. Size dependent strengthening in particle reinforced aluminium. Acta Materialia. 50(1), 39-51. https://doi.org/10.1016/S13596454(01)00327-5

[14] Lama D C C, Yang F, Chonga A C M, Wang J, Tong P, 2003. Experiments and theory in strain gradient elasticity. Journal of the Mechanics and Physics of Solids. 51, 14771508. https://doi.org/10.1016/S0022-5096(03)00053-X

[15] Li X B, Li L, Hu Y J, Ding Z, Deng W M, 2017. Bending, buckling and vibration of axially functionally graded beams based on nonlocal strain gradient theory. Composite Structures. 165, 250-265. https://doi. org/10.1016/j.compstruct.2017.01.032

[16] Liu Z, Meyers M A, Zhang Z, Ritchie R O, 2017. Functional gradients and heterogeneities in biological materials: Design principles, functions, and bioinspired applications. Progress in Materials Science. 88, 467-498. https://doi. org/10.1016/j.pmatsci.2017.04.013

[17] Ma H M, Gao X L, Reddy J N, 2008. A microstructuredependent Timoshenko beam model based on a modified couple stress theory. Journal of the Mechanics \& Physics of Solids. 56(12): 3379-3391. https://doi.org/10.1016/j. jmps.2008.09.007

[18] Mohammadabadi M, Daneshmehr A R, Homayounfard M, 2015. Size-dependent thermal buckling analysis of micro composite laminated beams using modified couple stress theory. International Journal of Engineering Science. 92, 47-62. https://doi.org/10.1016/j.ijengsci.2015.03.005

[19] Naebe M, Shirvanimoghaddam K, 2016. Functionally graded materials: A review of fabrication and properties. Applied Materials Today. 5, 223-245. https://doi. org/10.1016/j.apmt.2016.10.001

[20] Park S K, Gao X L, 2006. Bernoulli-Euler beam model based on a modified couple stress theory. Journal of Micromechanics \& Microengineering. 16(11), 2355-2359. http://iopscience.iop.org/ article/10.1088/0960-1317/16/11/015/meta

[21] Pradhan K K, Chakraverty S, 2013. Free vibration of Euler and Timoshenko functionally graded beams by Rayleigh-Ritz method. Composites Part B: Engineering. 51(4), 175-184. https://doi.org/10.1016/j. compositesb.2013.02.027

[22] Reddy J N, 2000. Analysis of functionally graded plates. International Journal for Numerical Methods in Engineering. 47(1-3), 663-684. https://doi.org/10.1002/ (SICI)1097-0207(20000110/30)47:1/3<663::AIDNME787>3.0.CO;2-8

[23] Reddy J N, 2011. Microstructure-dependent couple stress theories of functionally graded beams. Journal of the Mechanics \& Physics of Solids. 59(11), 2382-2399. https://doi.org/10.1016/j.jmps.2011.06.008

[24] Salamat-Talab M, Nateghi A, Torabi J, 2012. Static and dynamic analysis of third-order shear deformation FG micro beam based on modified couple stress theory. International Journal of Mechanical Sciences. 57(1), 6373. https://doi.org/10.1016/j.ijmecsci.2012.02.004

[25] Shahba A, Attarnejad R, Marvi M T, Hajilar S, 2011. Free vibration and stability analysis of axially functionally graded tapered Timoshenko beams with classical and non-classical boundary conditions. Composites Part B: Engineering. 42(4), 801-808. https://doi.org/10.1016/j. compositesb.2011.01.017

[26] Sola A, Bellucci D, Cannillo V, 2016. Functionally graded materials for orthopedic applications - an update on design and manufacturing. Biotechnology Advances. 34(5), 504-531. https://doi.org/10.1016/j. biotechadv.2015.12.013

[27] Thai H T, Vo T P, Nguyen T K, Lee J, 2015. Size-dependent behavior of functionally graded sandwich microbeams based on the modified couple stress theory. Composite Structures. 123, 337-349. https://doi.org/10.1016/j. compstruct.2014.11.065

[28] Thai H T, Vo T P, Nguyen T K, Kim S E, 2017. A review of continuum mechanics models for size-dependent analysis of beams and plates. Composite Structures. 177, 196- 
219. https://doi.org/10.1016/j.compstruct.2017.06.040

[29] Trinh L C, Nguyen H X, Vo T P, Nguyen T K, 2016. Size-dependent behaviour of functionally graded microbeams using various shear deformation theories based on the modified couple stress theory. Composite Structures. 154, 556-572. https://doi.org/10.1016/j. compstruct.2016.07.033

[30] Wu L, Wang Q S, Elishakoff I, 2005. Semi-inverse method for axially functionally graded beams with an anti-symmetric vibration mode. Journal of Sound and Vibration. 284(3-5), 1190-1202. https://doi. org/10.1016/j.jsv.2004.08.038

[31] Xu F, Zhang X, Zhang $H, 2018$. A review on functionally graded structures and materials for energy absorption.
Engineering Structures. 171, 309-325. https://doi. org/10.1016/j.engstruct.2018.05.094

[32] Yang F, Chong A C M, Lam D C C, Tong P, 2002. Couple stress based strain gradient theory for elasticity. International Journal of Solids and Structures. 39(10), 2731-2743. https://doi.org/10.1016/S0020-7683(02)00152-X

[33] Zdeněk $P$, Bažant, 1984. Size effect in blunt fracture: concrete, rock, metal. Journal of Engineering Mechanics. 110 (4), 518-535. https://doi.org/10.1061/(ASCE)07339399(1984)110:4(518)

[34] Zhao L, Chen W Q, Lü C F, 2012. Symplectic elasticity for bi-directional functionally graded materials. Mechanics of Materials. 54, 32-42. https://doi.org/10.1016/j. mechmat.2012.06.001 